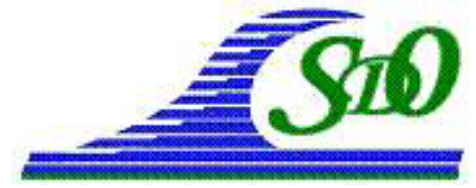

XI ìmes Journées Nationales Génie Côtier - Génie Civil

Les Sables d'Olonne, 22-25 juin 2010

DOI:10.5150/jngcgc.2010.027-D C Editions Paralia CFL

disponible en ligne - http://www.paralia.fr - available online

\title{
Méthode de développement intégré de modèles hydro-sédimentaires. Application au système estuarien de la Nivelle, France
}

\author{
Raphaëlle DORE $^{1}$, Philippe MARON ${ }^{1}$ \\ 1. Université de Pau et des Pays de l'Adour, LaSAGeC², 1 Allée du Parc Montaury, \\ 64600 Anglet, France. \\ raphaelle.dore@univ-pau.fr
}

\section{Résumé :}

Cet article présente une Méthode de Développement Intégré de modèles hydrosédimentaires. La première étape est la définition systématique et précise de la Problématique à laquelle doit répondre le modèle. La seconde étape est l'écriture du Cahier des Charges. Cette méthode permet de développer efficacement un modèle opérationnel répondant précisément à la Problématique. La méthode est appliquée au développement d'un modèle de transport des sédiments cohésifs dans l'estuaire de la Nivelle. Les résultats obtenus grâce à ce modèle couplé à un Plan d'Expériences permettent de définir les influences de la marée et du débit fluvial sur le budget sédimentaire dans le Port de St-Jean-de-Luz \& Ciboure.

\section{Mots-clés :}

Modèle numérique de transport - Analyse fonctionnelle - Cahier des charges

\section{Introduction}

La résolution de problématiques liées au transport sédimentaire nécessite fréquemment le développement de modèles numériques (CANCINO \& NEVES, 1999). Ceux-ci permettent notamment de simuler un nombre de scénarios hydro-climatiques que seules limitent les ressources temporelles et numériques. L'efficacité d'un modèle numérique repose tout d'abord sur un ensemble de choix de modélisation cohérents d'une Problématique posée. La qualité du modèle dépend entre autre des étapes successives de calibration et validation qui reposent sur la comparaison entre résultats de simulation et mesures in situ. Qualité et efficacité du modèle dépendent également des éventuelles optimisations, telles que le raffinement des résultats sur la zone étudiée. Comme le montre la figure 1, les phases de calibration, de validation et d'optimisation peuvent donner lieu à des boucles de retour sur les choix de modélisation, repoussant l'obtention de résultats exploitables. Dès lors, il apparaît nécessaire de proposer des outils qui permettent :

- de développer des modèles fonctionnels dont les résultats répondent précisément à une Problématique clairement définie au préalable ;

- de réduire le nombre de boucles retour sur les choix de modélisation en y intégrant les exigences liées à la calibration, à la validation et à l'optimisation. 


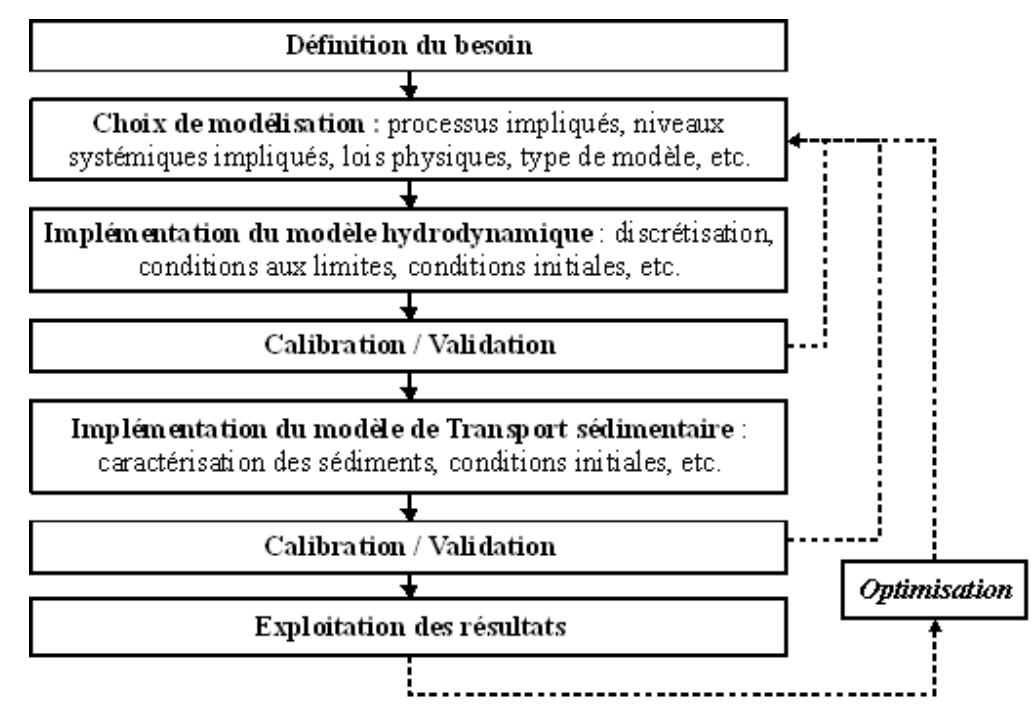

Figure 1. Processus de développement d'un modèle numérique.

\section{Méthode de Développement Intégrée de Modèle de Transport}

La Méthode de Développement Intégré de modèle Hydro-sédimentaire repose sur quatre étapes. Elle exploite les outils de l'Analyse Fonctionnelle et de la Méthode APTE® (BRETSCHE, 2000) utilisés pour la conduite de projets en Conception de Système.

\subsection{Définition du Besoin}

La première étape de cette méthode consiste à définir précisément la Problématique (ou Besoin) à laquelle le modèle doit répondre. L'outil méthodologique APTE $®$ dit diagramme de la "Bête à cornes" formalise les trois questions suivantes :

(a) A qui (quoi) le système rend-il service ?

(b) Sur quoi (quoi) agit-il ?

(c) Dans quel but?

Les réponses à ces trois interrogations en considérant la problématique formulée par un gestionnaire côtier ou portuaire, permet de formuler le Besoin sous la forme suivante : Sujet (le modèle développé) + Verbe(s) + Complément(s).

\subsection{Analyse systématique des Milieux extérieurs}

La seconde étape repose tout d'abord sur l'identification des différents contextes d'utilisation du modèle (Situations de Vie ou SdV) : simulation hydrodynamique, calibration et validation, etc. Pour chaque $\mathrm{SdV}$, le modèle est placé au centre des éléments qui constituent son Milieu Extérieur. Cet outil APTE® dit "diagramme Pieuvre" permet de mettre en évidence les éléments extérieurs qui contraignent le modèle (disponibilité des mesures, ressources, etc.) et de définir ainsi les fonctions Contraintes. La "Pieuvre" permet également d'identifier les relations existant entre des éléments extérieurs via le modèle et de formaliser les Fonctions Service. Au terme de 
cette étape le modélisateur connaît la liste exhaustive des fonctions auxquelles le modèle doit répondre.

\subsection{Etablissement du Cahier des Charges Fonctionnels (CdCF)}

La troisième étape de la méthode consiste à synthétiser, décrire et hiérarchiser l'ensemble des fonctions et contraintes dans un Cahier des Charges Fonctionnel (CdCF). $\mathrm{La}$ formalisation du $\mathrm{CdCF}$ amène à décomposer et préciser les fonctions. Un $\mathrm{CdCF}$ donne pour chaque fonction (BRETSCHE, 2000) :

(1) la définition : sous la forme sujet (le modèle) + verbe + complément ;

(2) le coefficient d'importance $\mathrm{K}$ de 1 (non négociable) à 5 (très négociable) qui permet de hiérarchiser les fonctions ;

(3) un (ou plusieurs) Critère(s) d'appréciation ou variable qui permet d'évaluer la réalisation de la fonction et qui peut être de type physique, floue, qualitative, etc.;

(4) un Niveau pour quantifier chaque Critère par une valeur, un intervalle, etc. ;

(5) la classe de Flexibilité F de 1 (non modulable) à 3 (très flexible) qui traduit la possibilité de moduler autour du niveau recherché.

\subsection{Choix de modélisation}

La quatrième étape est la recherche d'un ensemble de Fonctions techniques. Une Fonctions Technique est la solution technique ou numérique, qui réalise la Fonction Service correspondante. Le CdCF facilite la recherche de compromis entre les fonctions hiérarchisées. Par conséquent le CdCF facilite la recherche d'un ensemble de Fonctions Techniques qui synthétisent les caractéristiques de la problématique. La définition d'une Fonctions Technique nécessite parfois la décomposition systémique de la Fonction Service qu'elle réalise. Cette décomposition est facilitée par l'outil méthodologique FAST (Functional Analysis System Technic) (BRETSCHE, 2000). Les choix de modélisation correspondent aux Fonctions Techniques (Tab. 1, col. 6).

Lorsque le Besoin requiert la définition de scénarios impactant du point de vue de la problématique, nous préconisons l'utilisation des Plans d'Expériences. Ils permettent la mise en place d'une stratégie expérimentale correspondant à un ensemble de simulations numériques réalisées par le modèle. Mettre en place un Plan d'Expériences est une Fonctions Technique et influe sur les autres choix de modélisation. On peut décrire le domaine expérimental en travaillant aux extremums des intervalles décrits par les critères d'appréciation (variables physiques) pertinents de la Fonction Service réalisée. Ce type de Plan dit Factoriel $2^{\mathrm{k}}$ (deux niveaux et $\mathrm{k}$ critères) est souvent la stratégie expérimentale optimale (GOUPY, 2001).

\section{Application au modèle de transport sédimentaire dans l'estuaire de la Nivelle}

Le port de St Jean de Luz - Ciboure, à l'embouchure de la Nivelle, est soumis à des processus d'envasement. 


\subsection{Description du système estuarien de la Nivelle}

La Nivelle estuarienne est un système allongé et dominé par la marée (DORE et al., 2009). La profondeur moyenne du même ordre que l'amplitude moyenne de marée $(2.25 \mathrm{~m})$ induit des turbulences sur le fond qui sont suffisantes pour homogénéiser l'estuaire sur la verticale. Les courants de marée diminuant assez rapidement vers l'amont, les effets de la convergence des rives et des frottements des parois de l'estuaire sont opposés et équivalents (estuaire synchrone). Il est donc pertinent de négliger l'influence du vent à la surface libre devant les effets de la marée et du débit fluvial. L'influence de la houle sur le fond est faible en raison de l'absence de vagues significatives dans la baie protégée par trois digues. Les mesures de Concentration $C$ de sédiments en suspension $(1996,1997,2002)$ donnent une moyenne de l'ordre de $20 \mathrm{mg} \mathrm{L}^{-1}$ (DORE et al., 2009).

\subsection{Définition du Besoin}

L'application de la "Bête à Cornes" permet de formaliser le Besoin : Afin d'aider le Conseil Général des Pyrénées Atlantiques à gérer le dragage du port de St Jean de Luz \& Ciboure, le modèle Nivelle doit permettre de déterminer les scénarios impactant en termes de dépôt de sédiments cohésifs.

\subsection{Etablissement du Cahier des Charges Fonctionnels (CdCF)}

Le Tableau 1 montre une partie des contraintes et Fonctions Service pour trois Situations de Vie (Simulation hydrodynamique, Calibration de l'hydrodynamique, Simulation des Transports sédimentaires). Les Critères et les Niveaux correspondant reprennent la description de l'estuaire (gammes de débit, état du mélange vertical, etc.).

\subsection{Choix de modélisation}

Les choix de modélisation (Tab. 1, col. 6) synthétisent l'ensemble des caractéristiques du système estuarien de la Nivelle ainsi que l'influence des milieux extérieurs. Le modèle de type eulérien présente un maillage cartésien à pas variable (DORE et al. 2009).

Dans la Situation de Vie, calibration, une des fonctions techniques qui répond à la Fonction Service F_ch_1, préconise la pré-calibration de la viscosité numérique horizontale. La pré-calibration consiste à évaluer puis réduire l'intervalle des valeurs du critère de viscosité numérique horizontale. Ce critère d'appréciation de la fonction F_ch_1 a une grande influence sur les vitesses simulées. En effet la viscosité numérique horizontale est le principal paramètre sur lequel se joue la calibration hydrodynamique du modèle. En réduisant l'intervalle des valeurs susceptibles de convenir pour une calibration satisfaisante du modèle, nous réduisons le nombre de boucle retour sur les choix de modélisation. 


\section{XI $I^{\text {èes }}$ Journées Nationales Génie Côtier - Génie Civil Les Sables d'Olonne, 22-25 juin 2010}

Le choix de modélisation le plus important correspond à l'approche bidimensionnelle intégrée sur la hauteur d'eau, dit $2 \mathrm{DH}(H=\eta+h$ où $\eta$ est l'élévation de la surface libre et $h$ la profondeur). L'approche $2 \mathrm{DH}$ est fréquemment utilisée pour les estuaires bien mélangés et s'appuie sur les formes bidimensionnelles de l'équation de continuité (1) et des équations de la conservation de la quantité de mouvement (2).

$$
\begin{aligned}
& \frac{\partial \eta}{\partial t}+\frac{\partial(\eta+h) \cdot U_{1}}{\partial x_{1}}+\frac{\partial(\eta+h) \cdot U_{2}}{\partial x_{2}}=0 \\
& \frac{\partial U_{1}}{\partial t}+\frac{\partial U_{i} \cdot U_{1}}{\partial x_{i}}=f \cdot U_{2}-\frac{1}{\rho} \cdot \frac{\partial P}{\partial x_{1}}+v_{H} \cdot \frac{\partial^{2} U_{1}}{\partial x_{i}^{2}}+\frac{\tau_{1}^{S}-\tau_{1}^{F}}{\rho H} \text { et } \frac{\partial U_{2}}{\partial t}+\frac{\partial U_{i} \cdot U_{2}}{\partial x_{i}}=-f \cdot U_{1}-\frac{1}{\rho} \cdot \frac{\partial P}{\partial x_{2}}+v H \cdot \frac{\partial^{2} U_{2}}{\partial x_{i}^{2}}+\frac{\tau_{2}^{S}-\tau \tau_{2}^{F}}{\rho H}
\end{aligned}
$$

Tableau 1. Cahier des Charges Fonctionnel du Modèle de Transport dans l'estuaire de

\begin{tabular}{|c|c|c|c|c|c|c|}
\hline$S d V: S i n$ & nulation hydrodynamique & $K$ & Critère(s) & $\operatorname{Niveau}(x)$ & $F$ & Solutions techniques \\
\hline \multicolumn{7}{|c|}{ Fonctions Service (Echantillon des Fonctions de plus haute importance) : le modèle Nivelle... } \\
\hline$F \_s h \_l$ & $\begin{array}{l}\text { doit permettre la simulation de } \\
\text { l'écoulement en tenant compte du } \\
\text { fond }\end{array}$ & 5 & $\begin{array}{l}\text { - Bathymétries : océan, baie, } \\
\text { Nivelle (Port d'Ascain) } \\
\text { - Type de fond (Tau [Pa]) }\end{array}$ & $\begin{array}{l}\text { - Bathy disponibles du port } \\
\text { d'Ascain à l'océan } \\
\text { - Vase sur la zone d'intérêt }\end{array}$ & $\begin{array}{l}1 \\
3\end{array}$ & $\begin{array}{l}\text { - Tau défini par Formule de } \\
\text { Manning } \\
\text { - Coef de Manning par zone }\end{array}$ \\
\hline$F \_s h \_4$ & $\begin{array}{l}\text { doit permettre la simulation de } \\
\text { l'écoulement en tenant compte } \\
\text { des parois }\end{array}$ & 3 & - Pente de parois $[\mathrm{m} / \mathrm{m}]$ & $\begin{array}{l}\text { - Pentes douces sur estuaire et } \\
\text { verticale dans le Port }\end{array}$ & 2 & $\begin{array}{l}\text { Conditions de (non) } \\
\text { glissement }\end{array}$ \\
\hline$F_{-} s h \_7$ & $\begin{array}{l}\text { doit permettre la simulation de } \\
\text { l'écoulement en tenant compte du } \\
\text { débit (crue non débordante) }\end{array}$ & 5 & $\begin{array}{l}\text { - } Q[m 3 / s] \text { (hydreaufrance) } \\
\text { corrigé au seuil d'Ascain : étiage } \\
\text { à crue non débordantes }\end{array}$ & $\begin{array}{l}Q_{\text {etiage }}=3.7,55<Q_{2}<75 \\
100<Q_{10}<125,135<Q_{50}<160\end{array}$ & 2 & $\begin{array}{l}\text { Q imposé dans } 1 \text { maille } \\
Q_{\text {étiage: } 19 / 01 / 2009} \\
Q_{50:} 10 \text { au } 12 / 02 / 2009\end{array}$ \\
\hline$F_{-} s_{-} 9$ & $\begin{array}{l}\text { doit permettre la simulation des } \\
\text { écoulements en tenant compte de } \\
\text { l'état de mélange vertical }\end{array}$ & 4 & Stratification (0 ou 1) & o (bien mélangé) & 1 & Approche $2 D H$ \\
\hline \multicolumn{7}{|c|}{ Contraintes (Echantillon des Contraintes de plus haute importance) : le modèle Nivelle... } \\
\hline$C \_h \_l$ & $\begin{array}{l}\text { doit tenir compte des gammes de } \\
\text { courants réels (crue non } \\
\text { débordante) }\end{array}$ & 5 & - Vitesse max d'écoulement & $\begin{array}{l}-V_{\max }=1 \mathrm{~m} / \mathrm{s} \\
\text { (cf. ADCP de fév. 2009) }\end{array}$ & 2 & - Nombre de courant $C_{-} r$, \\
\hline$C \_h \_2$ & $\begin{array}{l}\text { ne doit pas être perturbé par la } \\
\text { limite d'influence océanique du } \\
\text { débit (crue non débordante) }\end{array}$ & 4 & - Influence du jet de la Nivelle & - digue de l'Artha $+/-20 m$ & 2 & $\begin{array}{l}\text { Taille de la bande côtière à } \\
\text { inclure dans le MNT (modèle } \\
\text { numérique de terrain) }\end{array}$ \\
\hline$C \_h \_3$ & $\begin{array}{l}\text { ne doit pas être perturbé par la } \\
\text { limite d'influence du flot dans la } \\
\text { rivière }\end{array}$ & 3 & $\begin{array}{l}\text { - Limite inversion de courant } \\
\text { - Limite influence en marnage }\end{array}$ & - Seuil d'Ascain $+/-1 \mathrm{~km}$ & 2 & $\begin{array}{l}\text { Longueur de la queue } \\
\text { d'estuaire dans le } M N T\end{array}$ \\
\hline$S d V: C a$ & libration hydrodynamique & $K$ & Critères & Niveau & $F$ & Solutions techniques \\
\hline \multicolumn{7}{|c|}{ Fonctions Service (Echantillon des Fonctions de plus haute importance) : le modèle Nivelle... } \\
\hline$F \_c h \_l$ & $\begin{array}{l}\text { doit permettre de simuler un } \\
\text { champ de vitesse bien corrélé } \\
\text { avec les mesures in situ }\end{array}$ & 4 & $\begin{array}{l}\text { - Nombre de mailles dans Port } \\
\text { - Disponibilité des mesures } \\
\text { - ARMAE }\end{array}$ & $\begin{array}{l}\text { - } 3 \text { mailles dans le chenal } \\
\text { - ADCP le 13.02.09 } \\
\text { - }[0.2 ; 0.5] \grave{a}+/-0.1 \text { près }\end{array}$ & $\begin{array}{l}2 \\
1 \\
2\end{array}$ & $\begin{array}{l}\text { - Maillage à } 8 \text { m dans le port } \\
\text { - champs de vitesse intégré } \\
\text { sur H } \\
\text { - viscosité numérique pré- } \\
\text { calibrée }\end{array}$ \\
\hline$F_{-} c h \_2$ & $\begin{array}{l}\text { doit permettre de simuler un } \\
\text { niveau d'eau bien corrélé avec } \\
\text { les mesures }\end{array}$ & 4 & $\begin{array}{l}\text { - Disponibilité des mesures } \\
\text {-ARMAE }\end{array}$ & $\begin{array}{l}\text { - Marégraphe de Socoa } \\
\text { - }[0.2 ; 0.4] \grave{a}+/-0.1 \text { près }\end{array}$ & $\begin{array}{l}1 \\
2\end{array}$ & $\begin{array}{l}\text { Distribution des Jauges de } \\
\text { marée } \\
\text { Mise en forme : série } \\
\text { temporelle }\end{array}$ \\
\hline$S d V: S i n$ & nulation des transports & $K$ & Critères & Niveau & $F$ & Solutions techniques \\
\hline \multicolumn{7}{|c|}{ Fonctions Service (Echantillon des Fonctions de plus haute importance) : le modèle Nivelle... } \\
\hline$F S \_s t \_3$ & $\begin{array}{l}\text { doit permettre la détermination } \\
\text { des scenarii impactant en terme } \\
\text { de budget sédimentaire }\end{array}$ & 5 & $\begin{array}{l}\text { - Expériences numériques } \\
\text { - débit et coefficient de marée }\end{array}$ & $\begin{array}{l}\text { - Plan d'Expériences } \\
\text { - extremum des facteurs }\end{array}$ & $\begin{array}{l}2 \\
2\end{array}$ & $\begin{array}{l}\text { - Plan Factoriel } 2^{2} . \\
-\{32 ; 106\} \text { et }\left\{Q_{\text {étiage }} ; Q_{50}\right\}\end{array}$ \\
\hline
\end{tabular}
la Nivelle (Echantillon des Fonctions et Contraintes de plus haute importance). 
Le modèle est forcé par le signal de marée (harmoniques via un réseau de jauges) à la frontière océanique et le débit fluvial (série temporelle des mesures à la station hydrologique de Cherchebruit et corrigées par la loi de Meyer) à la queue de l'estuaire. La viscosité cinématique turbulente horizontale $v_{H}$ est calculée par la loi de Kolmogorov sous l'hypothèse d'OZMIDOV (1990). La contrainte de cisaillement induite par le vent à la surface libre $\tau^{S}$ est négligée de même que celle induite par les vagues sur le fond. La contrainte de cisaillement au fond $\tau^{F}$ est donc induite par la vitesse d'écoulement et fonction du coefficient de Manning $n$ (3).

$\tau_{i}^{F}=\frac{g \cdot \rho \cdot n^{2}}{\sqrt[3]{H}} U i \sqrt{U_{1}^{2}+U_{2}^{2}}$

Le modèle de transport de sédiments est gouverné par l'équation d'advection-diffusion sur la concentration $C(4)$ avec $S_{E}$ le taux d'érosion défini d'après PARTHENIADES (1965) et $S_{D}$ le taux de dépôt défini d'après KRONE (1962).

$\frac{\partial H C}{\partial t}+\frac{\partial H U_{i} C}{\partial x_{i}}=\frac{\partial}{\partial x_{i}}\left(H D_{i j} \frac{\partial C}{\partial x_{i}}\right)+\left(S_{E}-S_{D}\right)$

Le taux d'érosion $S_{E}$ défini selon PARTHENIADES (1965) est non nul si $\tau^{F}>\tau_{E}=0.2 \mathrm{~N} \mathrm{~m}^{-2}$ (DYER, 1986). De même le taux de dépôt $S_{D}$ défini selon KRONE (1962) est non nul et les sédiments commencent à chuter vers le fond si $\tau^{F}<\tau_{D}=0.1 \mathrm{~N} \mathrm{~m}^{-2}$ (PARTHENIADES, 1965 ; DYER, 1986). A l'état initial le fond du modèle est recouvert d'une couche de sédiments $(5 \mathrm{~cm})$. Au terme d'une période d'établissement d'un mois et demi la concentration $C$ dans la colonne d'eau dépend des forçages hydrologiques du modèle et du flux de matière déchargée avec le débit fluvial.

\section{Implémentation et résultats}

\subsection{Calibration du modèle hydrodynamique}

La calibration du modèle 2DH de la Nivelle estuarienne repose essentiellement sur la paramétrisation de la viscosité turbulente horizontale $v_{\mathrm{H}}$. L'établissement du Cahier des Charges Fonctionnel, permet de réduire l'intervalle de valeurs dans lequel chercher la meilleure valeur de $v_{\mathrm{H}}$ pour ce modèle. On réduit ainsi le nombre de comparaison entre les mesures ADCP et les résultats de simulation reprenant la situation hydrologique du jour de mesure (DORE et al., 2009). Après calibration, les vitesses simulées et mesurées montrent une corrélation de bonne qualité (ARMAE $=0,3)$ selon la classification en fonction de l'ARMAE (5) proposée par SUTHERLAND et al. (2004). Le signal de marée simulé présente une excellente corrélation (ARMAE $=0,03$ ) avec le signal réel.

$A R M A E=\frac{|Y-X|-O E}{|X|}$

\subsection{Etude qualitative des effets de la marée et du débit sur le budget sédimentaire}

L'étude de l'influence de la marée et du débit sur le budget sédimentaire repose sur la mise en place d'un Plan d'Expériences de type factoriel $2^{2}$ (Tab. 2). Les séries 
temporelles de débit $Q$ et de concentration $C$ de sédiments en suspension sont tirées de mesures effectuées lors d'épisodes réels (DORE et al., 2009). Le tableau 2 donne le Bilan sédimentaire massique surfacique (BMS) pour trois jours d'imposition des conditions de marée et débit. La régression linéaire multiple des résultats de ce Plan d'Expériences permet de dégager la relation (6). Les coefficients sont significatifs pour un seuil de confiance à $95 \%$.

$$
B M S=-1.17-3.48 \times Q-2.43 \times \eta-1.55 \times Q \times \eta
$$

L'effet négatif (-2.43) de la marée sur le budget sédimentaire montre que le dépôt sédimentaire est d'autant plus important que l'amplitude de la marée est faible. De même l'effet du débit (-3.48) montre que plus le débit fluvial est faible, plus le dépôt sédimentaire augmente. En effet pour des marées de morte eau et/ou des débits d'étiage, les vitesses d'écoulement sont globalement plus faibles et les valeurs de contrainte de cisaillement $\left(\tau_{F}<\tau_{D}\right)$ propices au dépôt sont plus souvent atteintes. A l'inverse, pour des marées de vive eau et/ou des débits de crue, la contrainte de cisaillement au fond est plus élevée. Dans ce cas, les conditions de cisaillement propices à l'érosion $\left(\tau_{F}>\tau_{E}\right)$ s'imposent plus souvent. L'effet du débit (-3.48) (Tab. 2, ligne 6) est une fois et demi plus important que celui de la marée (-2.43). La figure 2 (zone draguée définie par polygone en pointillés) permet d'observer les zones du port qui sont particulièrement soumises aux effets de la marée et du débit. Il apparaît notamment que le débit de crue cinquantennale (Fig. 2, Exp. 2 \& 4) implique de l'érosion sur la globalité du port. L'interaction marée débit (Coefficient: -1.55) est importante (Fig. 2, Exp. 4). Cette relation statistiquement significative et physiquement cohérente des observations, permet de définir qualitativement les scénarios impactant en termes de budget sédimentaire.

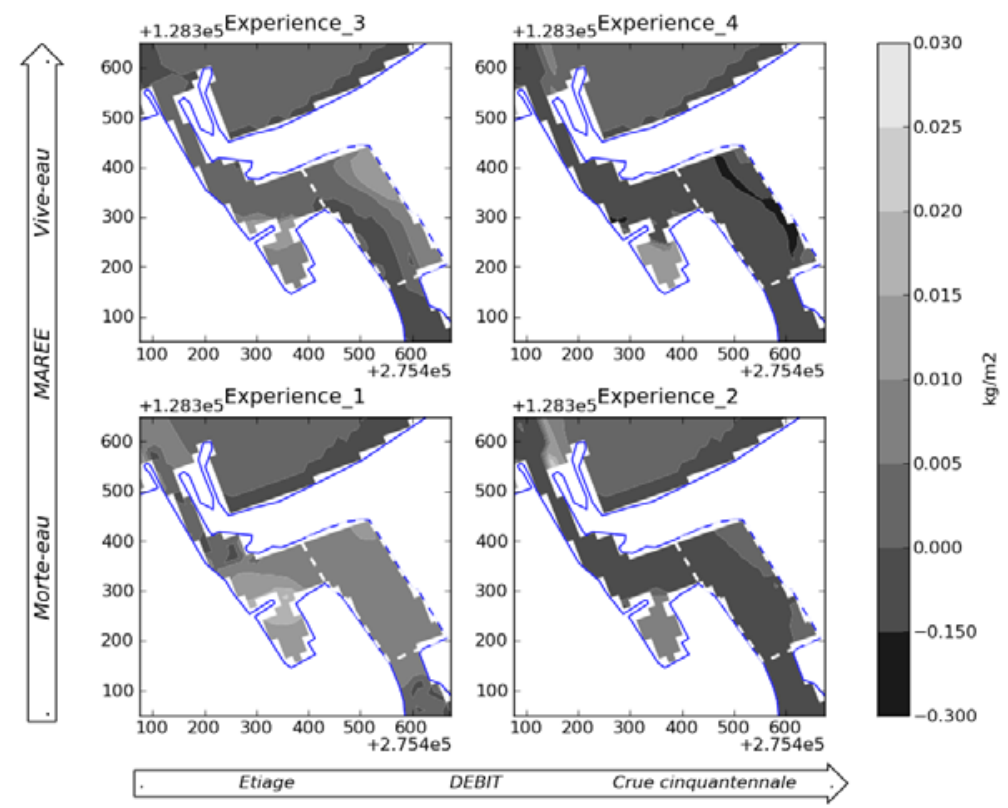

Figure 2. Budget sédimentaire $\left(\mathrm{kg} / \mathrm{m}^{2}\right)$ - expériences 1 à 4 (échelle non linéaire). 
Thème 2 - Dynamique sédimentaire et transports des particules

Tableau 2. Description des expériences numériques et des Budgets sédimentaires correspondants.

\begin{tabular}{lllll}
\hline Expérience & Conditions de marée & Conditions de débit & Nb d'Itérations & Bilan massique surfacique \\
\hline 1 & Marée de morte eau & Moyennes en étiage & 3 & $+3,23 \mathrm{~kg} / \mathrm{m}^{2}$ \\
& $1.91<\eta<3.25 \mathrm{~m}$ & $Q=3.71 \mathrm{~m}^{3} / \mathrm{s}$ et $C=7.10^{-3} \mathrm{~g} / \mathrm{L}$ & \\
2 & Marée de morte eau & Moyennes en crue quinquennale & 3 & $-0,63 \mathrm{~kg} / \mathrm{m}^{2}$ \\
& $1.91<\eta<3.25 \mathrm{~m}$ & $Q=142 \mathrm{~m}^{3} / \mathrm{s}$ et $C=3,5.10^{-3} \mathrm{~g} / \mathrm{L}$ & \\
3 & Marée de vive eau & Moyennes en étiage & 2 & $+1,46 \mathrm{~kg} / \mathrm{m}^{2}$ \\
& $0.5<\eta<4.65 \mathrm{~m}$ & $Q=3.71 \mathrm{~m}^{3} / \mathrm{s}$ et $C=7.10^{-3} \mathrm{~g} / \mathrm{L}$ & \\
4 & Marée de vive eau & Moyennes en crue quinquennale & 1 & $-8,59 \mathrm{~kg} / \mathrm{m}^{2}$ \\
& $0.5<\eta<4.65 \mathrm{~m}$ & $Q=142 \mathrm{~m}^{3} / \mathrm{s}$ et $C=3,5.10^{-3} \mathrm{~g} / \mathrm{L}$ & \\
\hline Effet & -2.43 & -3.48 & Interaction & -1.55 \\
\hline
\end{tabular}

\section{Conclusions et perspectives}

Le modèle Nivelle fournit la relation (6) qui permet de définir qualitativement les scénarios impactant en termes de budget sédimentaire. Par conséquent, ce modèle répond à la problématique énoncée précédemment. L'application de la Méthode de développement intégré permet de mettre en place des modèles plus rapidement calibrés. Ces modèles, grâce à de bons compromis entre précision et lourdeur numérique, peuvent être utilisés comme outil d'expérimentation numérique.

\section{Références bibliographiques}

BRETSCHE B. (2000). La méthode APTE. Ed. Pétrelle.

CANCINO L., NEVES R. (1999). Hydrodynamic and sediment suspension modelling in estuarine systems Part I \& II. Journal of Marine Systems, 22, pp 105-131. doi:10.1016/S0924-7963(99)00035-4

DORE R., MARON P., COYNEL A. (2009). Détermination des zones de dépôt / érosion des sédiments cohésifs à l'aide d'un Modèle de Transport 2DH. Congrès SHF.

DYER K.R. (1986). Coastal and estuarine sediment dynamics. Pub. Wiley-Interscience. GOUPY J. (2001). Introduction aux Plans d'Expériences. Ed. Dunod.

KRONE R.B. (1962). Flume studies of the transport of sediment in estuarial shoaling processes. Hydraulic Engineering Laboratory Report, Berkeley University.

OZMIDOV R.V. (1990). Diffusion of Contaminants in the Ocean. Oceanographic Sciences Library, Kluwer Academic Publishers, 283 p.

PARTHENIADES E. (1965). Erosion and deposition of cohesive soils. Journal of the Hydraulics Division, ASCE, vol. 91, pp 105-139.

SUTHERLAND J., WALSTRA D.J.R., CHESTER T.J., VAN RIJN L.C., SOUTHGATE H.N. (2004). Evaluation of coastal area modelling systems at an estuary mouth. Coastal Engineering $\mathrm{n}^{\circ}$ 51, pp 119-142. doi:10.1016/j.coastaleng.2003.12.003 